\title{
FAMILIAL SYRINGOMYELIA WITH MENTAL IMPAIRMENT
}

\author{
CAPT T O JEFFERSON, MD, RAMC \\ British Military Hospital, Hong Kong \\ DR C COHEN, FRCP (Ed and Glas) \\ Stracathro Hospital, Brechin, Angus
}

SUMMARY: The occurrence of an example of familial syringomyelia is reported and, for the first time in the literature, associated with mental impairment. The latter has ranged from simplicity to psychosis in different members of the family.

\section{Introduction}

A review of the world literature on the incidence of familial syringomyelia reveals that only three such cases have been described outside the United Kingdom, and only two in the United Kingdom ${ }^{1}$. However, as suggested by Bentley et al, familial syringomyelia may be less rare than the number of reported cases would suggest. Two cases are now reported from the Stracathro Hospital.

Case 1

\section{Case Reports}

Mr J S, aged 67 years, was admitted to the Assessment Unit for rehabiliation following a particularly severe fall. He had a 48 year old history of progressive muscle weakness starting in the right upper limb and then gradually extending to involve his right lower limb, and to a lesser extent, his left side, with progressive severe kyphoscoliosis. His disability eventually became so great that he had to retire from work while still in his 40s. At home he had sustained repeated falls over a number of years.

\section{On examination}

He had a marked kyphoscoliosis and a short neck with a strawberry coloured mark at the back. X-ray examination of his skull and spine showed "small osteoma in the frontal sinus", a "fairly high odontoid peg in the lateral view" and a "dorsal kyphoscoliosis" with "osteoporosis". A CAT scan showed "minimal cerebral atrophy, but no other relevant abnormality". These examinations were greatly impaired by the inability to position the patient's head conveniently due to his scoliosis.

The patient was referred to a Consultant Neurologist, who reported that "there was marked kyphoscoliosis and wasting and fasciculation in the right arm, chest muscle, triceps, deltoid, rhomboids, brachio-radials and that there was weakness to a greater extent on the right than the left. There was a fixed flexion deformity in the right hand, and impaired finger movement in the left hand. Tendon reflexes were absent in the upper limbs. In the legs, tendon reflexes were present, power was normal, tone was difficult to evaluate because of failure of relaxation, but there was a suggestion of an increase. The plantar responses were flexor. There was no cranial nerve or optic nerve abnormalities." 
Case 2

Miss A S, aged 74 years and the elder sister of $J$ S, also had a long history of progressive disability, numerous fractures caused by repeated falls and had been in Continuing Care in the Department since 1969.

\section{On examination}

She had a marked Kyphoscoliosis. An X-ray taken in 1966 suggested a hemi-vertebra in the dorsal region.

The patient was referred to the Consultant Neurologist. He reported that "she had a gross kyphoscoliosis to the right. There was marked wasting, weakness and some fasciculation in the right upper limb and some weakness in the left upper limb, but less wasting and no fasciculation. In the legs there was a slight reduction in power in the flexor groups, the tone was slightly increased and the plantar responses were extensor. Sensation was difficult to assess, but there was definite abnormality, in particular concerning pain sensation in the upper limbs. There were minor rapid movements of the fingers seen occasionally, choreiform in type."

Limited nerve conduction and EMG studies were carried out, again by the Consultant Neurologist, on both patients and "both show slowing of median nerves, but retention of ulnar nerve conduction, but PS showed slowing of common peroneal conduction". He thought that the likeliest diagnosis would be syringomyelia.

\section{Discussion}

Both patients have features suggestive of syringomyelia, although some of the tests carried out were impaired either by their physical disability or by their difficulty in concentrating. They both show some degree of mental retardation, especially in the case of $\mathbf{J} \mathbf{S}$.

Enquiries made about the ascendants of $\mathrm{J} S$ and $\mathrm{A} S$ indicate that their mother, ES was known to have a marked spinal deformity and mental simplicity, although this information is based on the recollections of people who knew her. There is no medical record of her, and she died in 1963 at the age of 80 . One other sister of JS and AS was apparently physically normal, although she had a long history of mental illness.

In the two cases presented, although a Chiari I malformation, with or without herniation of cerebellar tonsils and spina bifida ${ }^{1}$ is absent, evidence of a degree of spina bifida is present in the case of A S.

\section{Acknowledgements}

Dr D L W Davidson, Consultant Neurologist, Ninewells Hospital, Dundee, kindy carried out the detailed neurological examination.

\section{REFERENCE}

1 Bentley S J, Campbell, M J and Kaufman, P. Familial syringomyelia. $J$ Neurol Neurosurg Psychiatry 1975; 38: 346-349. 